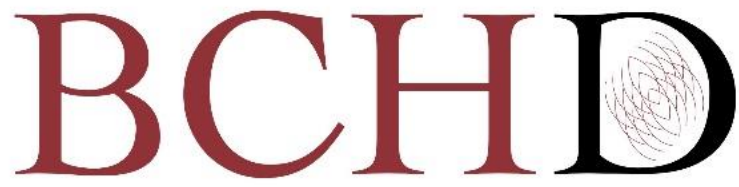

Bioactive Compounds in Health and Disease

\title{
Aging and chronic illnesses: Membrane Lipid Replacement for restoring mitochondrial function and reducing fatigue, pain, and other symptoms in aged individuals
}

\author{
Garth L. Nicolson ${ }^{1 *}$, Paul C. Breeding ${ }^{2}$, Robert Settineri ${ }^{3}$, and Gonzalo Ferreira de Mattos ${ }^{4}$
}

${ }^{1}$ Department of Molecular Pathology, The Institute for Molecular Medicine, Huntington Beach, California 92649, USA; ${ }^{2}$ Blue Hole Chiropractic and Wellness, Houston, Texas 77035, USA; ${ }^{3}$ Sierra Productions Research, Irvine, California 92692, USA; ${ }^{4}$ Laboratory of Ion Channels, Biological Membranes and Cell Signaling, Department of Biophysics, Facultad de Medicina, Universidad de la República, Montevideo, Uruguay

*Corresponding author: Prof. Emeritus Garth L. Nicolson, PhD, MD (H), Department of Molecular Pathology, The Institute for Molecular Medicine, P.O. Box 9355, S. Laguna Beach, CA 92652; USA

Submission Date: September 13 ${ }^{\text {th }}, 2020$; Acceptance Date: October 23 ${ }^{\text {rd }}, 2020$; Publication Date: October $28^{\text {th }}, 2020$

Please cite this article as: Nicolson G., Breeding P., Robert S., Ferreira de Mattos G. Aging and chronic illnesses: Membrane Lipid Replacement for restoring mitochondrial function and reducing fatigue, pain, and other symptoms in aged individuals. Bioactive Compounds in Health and Disease 2020. 3(10): 194-203. DOI: https://www.doi.org/10.31989/bchd.v3i10.749

\begin{abstract}
Membrane Lipid Replacement (MLR) is the use of functional dietary supplements containing cell membrane glycerolphospholipids and antioxidants to safely replace and remove damaged essential membrane phospholipids that accumulate during aging and in various chronic and acute illnesses. Most, if not all, clinical conditions and aging are characterized by cellular membrane phospholipid free radical oxidative damage, resulting in loss of membrane
\end{abstract}

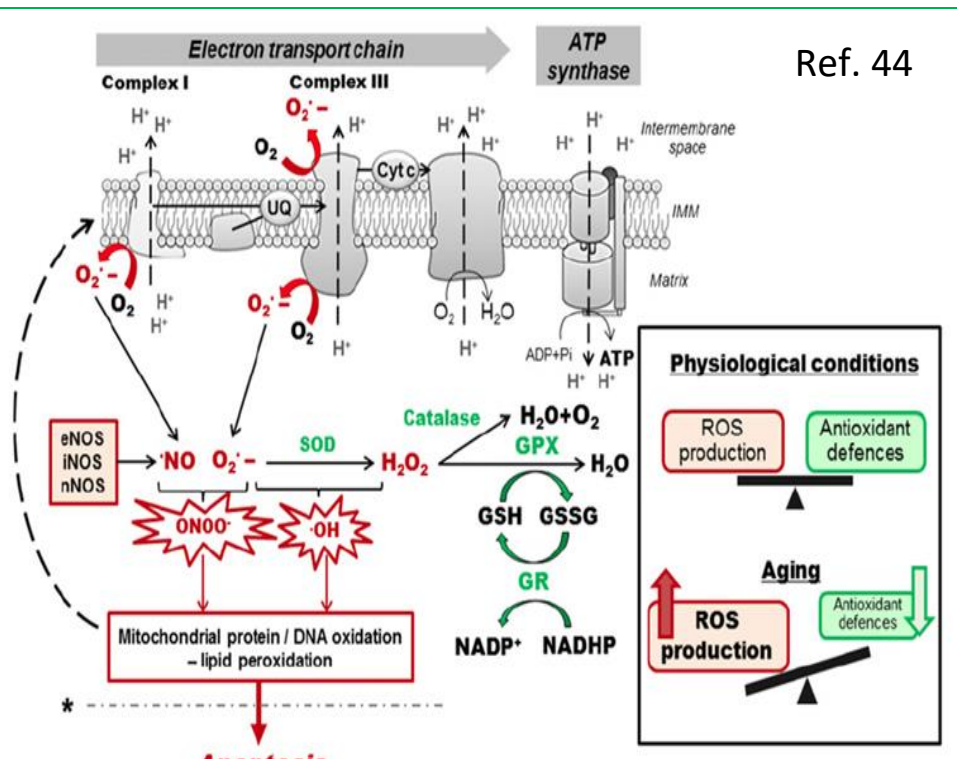

Apoptosis 
and cellular functions. In particular, loss of mitochondrial function, the key organelle responsible for over $90 \%$ of cellular energy production, can result in excess fatigue and other symptoms, and this is a common problem in almost all, if not all, age-related chronic diseases. Clinical trials have shown the benefits of MLR supplements in replenishing damaged membrane phospholipids and restoring mitochondrial function, resulting in reductions in fatigue and other symptoms in aged subjects and patients with a variety of clinical diagnoses. Here we have specifically reviewed the beneficial results of MLR on subjects older than 60 years. MLR provides general membrane and nutritional support during aging and illnesses to improve membrane function and overall health without risk of adverse effects. The case reports here and elsewhere and published clinical trials demonstrate that this is a safe and effective alternative or addition to pharmaceutical approaches for alleviating fatigue, pain, gastrointestinal and other symptoms associated with normal aging and age-related chronic illnesses.

Keywords: Aging, Fatigue, Pain, Gastrointestinal symptoms, Phospholipids, Clinical trials, Case reports, Cellular membranes

CFFC 2020. This is an Open Access article distributed under the terms of the Creative Commons Attribution 4.0 License (http://creativecommons.org/licenses/by/4.0)

\section{INTRODUCTION}

During aging and in essentially all chronic and many acute illnesses critical membrane phospholipids and other cellular components are damaged by excess free radical oxidants [1-5]. The free radical damage that is inflicted on cellular structures has been proposed to be the cause of aging $[3,4]$. But it is now accepted to be unlikely as a sole cause of aging and disease $[6,7]$. However, it is at least importantly involved along with other mechanisms in the aging process $[5,6]$ as well as disease processes $[7,8]$.

When membrane phospholipids that form the matrix of all biological membranes [9] are damaged, they must be repaired or replaced to return cellular functions to normal $[10,11]$. Thus the replacement of damaged membrane phospholipids with undamaged, functional membrane phospholipids is important for a variety of cellular and tissue functions and for general health [10-14]. For example, the membrane phospholipids that form the plasma membrane and intracellular membranes provide separation of enzymatic and chemical reactions into discrete cellular compartments or organelles. They are also essential for the function of membrane enzymes. In addition, they are an important energy storage reservoir and provide precursors for bioactive molecules that function in signal transduction and molecular recognition pathways [9-11]. Thus membrane phospholipids are essential for general health, and they must be constantly replaced in order to maintain well being [10-14].

The simplist way to maintain healthy cellular membranes is an appropriate diet [10-13]. However, since this often can't be achieved, dietary supplements containing membrane phospholipids have been used to maintain cellular membrane health [10-13]. Orally ingested phospholipids can be split into degradation products within the gastrointestinal system and absorbed; or they can be absorbed and taken in as intact molecules without degradation $[10,11]$. When present in excess in the gastrointestinal system, they can be absorbed as small phospholipid globules and micelles with undegraded constituents (reviewed in [11]). 
Independent of the transport mechanism, the process is very efficient-over $90 \%$ of ingested phospholipids are absorbed and transported into the blood within six hours [15-17]. In the blood circulation phospholipids are usually found in carrier molecules, such as lipoproteins, or cell membranes of erythrocytes; but in excess they can also be present as small phospholipid globules or micelles. Eventually they are delivered to tissues and cells where they are transferred by direct membrane contact, endocytosis or by carrier and transport proteins into cells where they are directed to various cellular and organelle membranes and compartments [11, 15-17].

The major membrane phospholipids, such as the glycerolphospholipids, can be enzymatically modified during their delivery, for example by substitution of their fatty acid side chains, to reflect the specific compositions of the membranes at their final destination. Once they have been partitioned into their terminal membrane sites, glycerolphospholipids and other membrane phospholipids can be further enzymatically modified to reflect the changing needs of cells . The entire process of uptake, transport, replacement and removal is driven by mass action, so when in great excess, intact phospholipids have an advantage in being able to reach their final cellular destination without significant enzymatic degradation or free radical modification. Also, the removal of damaged, oxidized phospholipids is also a mass action process (reviewed in [11]).

Patients with chronic illnesses as well as aged individuals are often deficient in undamaged MLR phosolipids, because dietary sources usually cannot provide enough MLR lipids for maintance of undamaged cellular membranes [11]. Thus health can be impaired. To provide the necessary MLR glycerolphospholipids these lipids have been used as dietary or therapeutic treatments or as supporting nutrients for health maintainance $[10-13,18,19]$.

\section{APPLICATIONS OF MEMBRANE LIPID REPLACEMENT TO AGING}

Although membrane lipids are mostly used for general health $[12,19]$, the most common therapeutic use of MLR is to treat fatigue $[10,11,13]$. Fatigue is the most common complaint of patients seeking general medical care, and it is associated with aging and most if not all chronic medical conditions [20]. Fatigue is considered a complex, multidimensional sensation that is not well understood but is perceived to be associated with a loss of overall energy, mental or physical tiredness, a feeling of exhaustion or diminished endurance, and an inability to perform even simple tasks without exertion [20, 21]. Fatigue develops in aged idividuals and in chronic diseases due to a variety of causes, including loss of mitochondrial function [21, 22]. Moderate to severe fatigue has been directly related to loss of mitochondrial function and diminished production of ATP by mitochondria [21, 22].

In some chronic clinical conditions fatigue is the most recognizable symptom - for example, in chronic fatigue syndrome/myalgic encephalomyelitis (CFS/ME) [23, 24]. Fatigue, however, is a rather general symptom, and it is found in many chronic illnesses and in normal aging [20]. Even in certain psychiatric diseases, such as schizophrenia, bipolar disease, among others, some cancers and other conditions, fatigue is among the most common symptom found, and in these cases it may or may not be associated with mitochondrial dysfunction [11, 22]. As an example, depression in some psychiatric disoders and in cancer patients is frequently related to mild fatigue states that show some physical, 
cognitive and emotional overlap with fatigue found in fatiguing illnesses [25].

MLR has been successfully used for reducing fatigue in patients with chronic fatigue (fatigue lasting more than 6 months), chronic illnesses, such as CFS/ME, fibromyalgia, and other fatiguing illnesses $[11,13,26-28]$. In a cross-over MLR clinical study on the effects of oral NTFactor ${ }^{\oplus}$ (a mixture of glycerolphospholipids, pre- and probiotics and other ingredients $[11,13])$ on chronic fatigue in moderately to severely fatigued subjects aged 60 years and older (61-77 years-old, average age $=68.9$ years-old), there was good correspondence between reductions in fatigue and improvements in mitochondrial function, as assessed by mitochondrial inner membrane transmembrane potential using a redox dye and fluorencence-activated cell sorting to examine individual peripheral blood leukocytes [26]. After 8 weeks on the MLR supplement containing $4 \mathrm{~g}$ per day of NTFactor ${ }^{\circledR}$ mitochondrial function improved significantly $(26.8 \%$ increase, $p<0.001)$ and fatigue was reduced significantly ( $33 \%$ decrease, $p<0.001$ ), and after 12 weeks of NTFactor ${ }^{\circledR}$, mitochondrial function increased by $35.5 \%(p<0.001)$ and found to be similar to the levels found in young healthy adults aged 30 years-old, and fatigue was reduced by $35.7 \%$, $p<0.001$ [26]. After 12 weeks of oral NTFactor supplementation, the subjects were placed on placebo without their knowledge for an additional 12 weeks, and their fatigue and mitochondrial function levels were measured. After crossing over to the 12week placebo period, fatigue and mitochondrial function were intermediate between the initial starting values and those found after eight or 12 weeks on the MLR supplement [26]. This study indicated that aged individuals with moderate to severe chronic fatigue benefited significantly from taking the oral MLR supplement, and their mitochondrial function, as measured by the inner mitochondrial inner membrane trans-membrane potential, was similar to much younger subjects, but only if they continued to take the oral MLR supplement.

Similar positive results on the effects of the MLR supplement NTFactor ${ }^{\circledR}$ on reducing fatigue were found in patients with chronic fatigue syndrome (CFS/ME), fibromyalgia, Gulf War illness and chronic Lyme disease (reductions in fatigue of $26-43 \%$ ) [11, $13,27]$. In some of these studies the effects of oral MLR with NTFactor can be compared between the younger subjects ( $<60$ years-old) and older subjects (>60 years-old). Both the younger and older subjects benefited significantly from the MLR supplement NTFactor ${ }^{\oplus}$. Although there were slight improvements in fatigue scores in the older subjects (>60 years-old) compared to those under the age of 60 years on NTFactor ${ }^{\oplus}$, statistical comparisons of the older-vyounger results showed that the differences did not reach statisical significance (data not shown). This indicates that with respect to fatigue scores older persons benefit at least as much as younger persons when taking the oral MLR supplement.

In aged subjects MLR phospholipids have also been used to treat memory loss. For example, in a clinical study that used aged subjects and patients with Alzheimer's disease participants received daily supplementation of $300 \mathrm{mg}$ of phosphatidylserine for 6 months [29]. At the end of this period participants in the study demonstrated significant cognitive improvements relative to placebo controls [29]. This result, however, was not confirmed in another study on elderly subjects with age-associated memory impairment that received $300-600 \mathrm{mg} /$ day soy phosphatidylserine for the much shorter period of 12 weeks [30]. Improvements in mental clarity while on MLR phospholipid supplements have been examined 
in a study using NTFactor Lipids`. Twenty-nine subjects were given $0.6 \mathrm{~g}$ of NTFactor Lipids ${ }^{\circ}$ in a drink, and fatigue and mental focus were surveyed after three hours using a self-reported survey instrument. A majority of the participants responded within 1 hour, and by 3 hours improvements in cognition, mental clarity and focus along with reductions in perceived fatigue were reported [31].

In addition to fatigue and mental clarity, MLR supplements, such as NTFactor Lipids, have also been used to reduce pain, gastrointestinal and other symptoms in chronic illness patients. For example, in fibromyalgia patients $4.8 \mathrm{~g}$ per day of oral NTFactor Lipids $^{\circ}$ for 8 days reduced signficiantly pain $(p<0.001)$, fatigue $(p<0.001)$, gastrointestinal symptoms $(p<0.001)$ and improved Quality of Life assessments $(p<0.001)$ in an open label clinical trial [32]. This has now been followed by initiating an $\mathrm{NIH}$-approved (NCT03288389) randomized, placebo-controlled cross-over trial. Unfortunately, due to COVID-19 the results of this trial were not available at the time of drafting of this contribution. We plan to continue this trial when the interference of COVID-19 can no longer affect the results.

\section{APPARENT DOSE-RESPONSE OF MEMBRANE LIPID} REPLACEMENT

Over the several years of development of MLR supplements like NTFactor ${ }^{\circ}$ and NTFactor Lipids ${ }^{\circ}$ the amounts of suggested daily doses for health or symptoms have changed or evolved, depending on the result that was desired or anticipated (for example, see Table 2 of ref. 13). In our initial studies where a blend of NTFactor ${ }^{\circ}$ containing pro- and prebiotics and other ingredients was used, the intent was to provide a dose of MLR phospholipids that would result in eventual turnover of damaged mitochondrial and other membrane phospholipids and incorporation of undamaged, functional phospholipids in order to increase function to patients or to the elderly. In particular, our initial aim was to repair enough damage to mitochondrial membranes to return mitochondrial function to the levels seen in normal age-matched adults. We were less concerned with the time required to make this happen, or to achieve maximal dose-responses. Although we have not tested enough subjects to establish an actual dose-response relationship between mitochondrial function and MLR glycerolphospholipid daily oral doses, we have found that use of lower doses of membrane phospholipids (for example, 0.7-1 g per day NTFactor Lipids ${ }^{\circ}$ ) can be effective eventually. However, this generally required longer periods of time, effectively several weeks to months. This time may be necessary to turn over membrane stores, remove damaged membrane phospholipids and replace them with undamaged phospholipids. We have found that higher doses (4-5 g per day NTFactor Lipids ${ }^{\circ}$ ) achieve similar reductions in symptoms, such as fatigue scores, but does this more quickly, in several days to a week or more instead of several months [32, 33].

To resolve some symptoms higher minimum doses of MLR, above several g ( $>4$ ) per day, have been used $[32,33]$. Thus in our recent work on widespread pain we have found that robust responses or large reductions in pain and other symptoms require higher doses per day of oral MLR glycerolphospholipids to be effective, and many patients themselves gradually gravitate to higher daily oral doses of MLR phospholipids (5-6 g or more per day NTFactor Lipids ${ }^{\circ}$ ). One possible approach to the continued use of high levels of MLR phospholipids could be to initially use a loading dose of several g (4-6) per day of MLR phospholipids and then lower the daily dose 
to a more moderate maintainance dose (2-3 g per day), depending on the desired outcome.

\section{APPLICATIONS OF MEMBRANE LIPID REPLACEMENT}

\section{TO AGING: CASE STUDIES}

We have seen the positive effects of NTFactor Lipids ${ }^{\bullet}$ in aged patients who have widespread musculoskeletal pain and chronic fatigue. For our purposes the descriptions of the effects of MLR with NTFactor Lipids ${ }^{\circledR}$ on aged patients are abbreviated. Only the most pertinent and brief descriptions of the results in a few patient examples are given below. These new case reports add to existing case reports on reduction of pain and fatigue by use of oral NTFactor Lipids ${ }^{\circledR}[33]$.

Patient A: Patient $A$ is a 63 year-old man who presented with chronic fatigue, severe musculoskeletal pain, vision loss, memory loss, constipation, gastrointestinal pain, sleep difficulties and other signs and symptoms. He began MLR with 4 g oral NTFactor Lipids ${ }^{\oplus}$ powder disolved in a glass of water each morning. After 2 weeks he reported that his gastrointestinal pain and constipation had resolved, and he reported less fatigue and some reductions in musculoskeletal pain. He then increased his daily dose of NTFactor Lipids ${ }^{\circledR}$ powder to $6 \mathrm{~g}$ per day. By 6 weeks his chronic fatigue, musculoskeletal and gastrointestinal pain were greatly reduced, although not fully resolved, and he reported improvements in vision and cognition, although these were not independently varified. He is now using $6 \mathrm{~g}$ per day NTFactor Lipids ${ }^{\circledR}$ powder to maintain the improvements he has achieved, and he continues to see improvements in pain, memory loss and gastrointestinal pain and constipation. He also reports that his sleep difficulties have basically resolved.
Patient B: Patient B is a 68 year-old post-menopausal woman who suffered from widespread musculoskeletal pain, chronic fatigue, hair loss, sleep problems and severe headaches. She started taking $4.8 \mathrm{~g}$ NTFactor Lipids $^{\circledR}$ in chewable wafers, and within one week reported less musculoskeletal pain, chronic fatigue and headaches. After 3 weeks she reported further improvements in pain and fatigue, and her headaches and sleep problems had continued to improve. She has reported continued improvements in fatigue and musculoskeletal pain and headaches over a 6-week period. She did not increase the dose of NTFactor Lipids in chewable wafers beyond $4.8 \mathrm{~g}$ per day.

Patient C: Patient C, a male of age 59 years, was a disabled veteran of the first Gulf War (1991). Since his return from Kuwait, he had suffered from chronic fatigue and widespread pain, and he had been treated with cognitive therapy, narcotics and antidepressants for years without lasting relief from his symptoms. At the time of presentation, he had stopped all medications and complained of widespread musculoskeletal pain, chronic fatigue, headaches, myalgia, arthralgia, and skin lesions. His diagnoses were Gulf War Illness and fibromyalgia. He started MLR with $4 \mathrm{~g}$ per day NTFactor Lipids ${ }^{\circledR}$ wafers and after approximately one week he increased the dose to $5 \mathrm{~g}$ per day NTFactor Lipids ${ }^{\circledR}$ wafers. Within 2 weeks of MLR he reported improvements in fatigue and musculoskeletal pain severity, along with reductions in the frequency and severity of headaches. Within an additional week of MLR his sleep problems also improved, and he was able to sleep a full night for the first time in more than a decade. He continued on the oral MLR supplement for at an additional 2 months at $6 \mathrm{~g}$ per day, and his 
condition has continued to slowly improve. Although he has not reported full recovery from his condition, he reported that his symptoms have been greatly reduced in severity, and he is anticipating eventually going back to work.

\section{DISCUSSION}

MLR supplements containing NTFactor ${ }^{\circledR}$ blend or NTFactor Lipids ${ }^{\circledR}$ have been used as anti-aging supplements to help aged subjects decrease their age-associated symptoms and improve their quality of life $[10,11,13,26,34]$. In addition to fatigue, pain and other symptoms associated with aging, MLR supplementation appears to be useful for delaying some of the functional decline seen in patients as they age $[13,26,34,35]$. For example, some elements of functional decline associated with aging appear to be susceptible to MLR supplementation, and this is associated with particular morbidities, such as coronary heart disease, stroke, neurodegeneration and metabolic disorders [15, 29, 30, 34, 35]. For example, the most common morbidities associated with aging in North America are coronary heart disease and stroke, which have been, in turn, related to the blood levels of a marker, homocysteine [36, 37]. Patients at risk for coronary heart disease and stroke have elevated blood homocysteine levels, and a group of such patients were placed on oral supplementation with NTFactor ${ }^{\bullet}$ blend containing NTFactor Lipids ${ }^{\circledR}$, vitamins and minerals [38]. Within 6 months their blood homocysteine levels went from high risk levels (mean,10.85 $\pm 0.42 \mu$ mole/L) to blood levels well below (mean, $7.40 \pm 0.42 \mu \mathrm{mol} / \mathrm{L}$ ) those levels that predict hospitalization and death due to coronary disease [38].

Here patients taking an oral MLR supplement containing NTFactor Lipids showed reductions in fatigue scores but also reductions in pain and other symptoms. Pain is a complex symptom involving nerve membrane channelopathies, autoimmune responses and other factors $[39,40]$. Thus NTFactor Lipids may not act solely by replacement of damaged nerve or mitochondrial membrane glycerolphospholipids, but also by modulating and restoring the functions of cell membrane ion channels, intracellular $\mathrm{Ca}^{2+}$ concentrations as well as other properties (example, ref. [41]). Indeed, the main nerve cell membrane ion channels related to pain pathways (TRPV channels) can be dramatically modulated in their function by particular membrane phospholipids [42]. In addition, other properties, such as stimulation of free radical oxidative damage or the presense of proapoptotic enzymes like caspases, could be modulated by MLR phospholipids [43].

Future studies will initiate and concentrate on sensory properties, cognition, memory loss and other factors associated with loss of quality of life in the aged population.

List of Abbreviations: Chronic fatigue syndrome/myalgic encephalomyelitis, CFS/ME; MLR, Membrane Lipid Replacement; TRPV, transient receptor potential channels of the vanilloid subtype;

Disclosures: Garth L. Nicolson is a part-time consultant to Naturally Plus Tiawan and Nutritional Therapeutics, Inc. Robert Settineri is a part-time consultant to Naturally Plus USA and Nutritional Therapeutics, Inc. Paul Breeding and Gonzalo Ferreira de Mattos have no conflicts to disclose.

Acknowledgements and Contributions: The authors would like to thank Nutritional Therapeutics, Inc. and the Institute for Molecular Medicine for financial support and for providing study materials. The article was written by Garth L. Nicolson with the input of the 
other authors. Garth Nicolson and Paul Breeding contributed patients to the case studies.

\section{REFERENCES:}

1. Knight JA: Diseases related to oxygen-derived free radicals. Annuals of Clinical and Laboratory Science 1995, 25(2): 111-121.

2. Gutteridge JMC: Free radicals in disease processes: a compilation of cause and consequence. Free Radical Research Communications 1993, 19(3): 141-158. doi/pdf/10.3109/10715769309111598?needAccess=true

3. Harman D: Aging: a theory based on free radical and radiation chemistry. Journal of Gerontology 1953, 11(3), 298-300.

doi.org/10.1093/geronj/11.3.298

4. Harman D: Aging: Free radical theory of aging. Mutation Research/DNAging $\quad 1992, \quad 275(3): \quad$ 257-266. doi:10.1016/0921-8734(92)90030-s

5. Aruoma Ol, Kaur H, Halliwell, B: Oxygen free radicals and human diseases. Journal of the Royal Society of Health 1991, 111(5): 172-177. doi/10.1177/146642409111100506

6. Viña, J, Borras, C, Abdelaziz KM, Garcia-valles R, GomezCabrera M: The free redical theory of aging revisited: the cell signaling disruption theory of aging. Antioxidants \& Redox Signaling 2013, 19(8): 779-787. doi.org/10.1089/ars.2012.5111

7. Ghezzi P, Jaquet V, Marcucci F, Schmidt HHW: The oxidative stress theory of disease: levels of evidence and epistemological aspects. British Journal of Parmacology 2017, 174: 1784-1796. doi/epdf/10.1111/bph.13544

8. Adibhatla RM, Hatcher JF: Lipid oxidation and peroxidation in CNS health and disease: from molecular mechanisms to therapeutic opportunities. Antioxidants and Redox Signaling 2010, 12: 125-169. doi.org/10.1089/ars.2009.2668

9. Nicolson GL: The Fluid-Mosaic Model of Membrane Structure: still relevant to understanding the structure, function and dynamics of biological membranes after more than 40 years. Biochimica et Biophysica Acta Biomembranes 2014, 1838: 1451-1466.

doi.org/10.1016/j.bbamem.2013.10.019

10. Nicolson GL: Membrane Lipid Replacement: clinical studies using a natural medicine approach to restoring membrane function and improving health. International Journal of Clinical Medicine 2016, 7: 133-143.

DOI: $10.4236 / \mathrm{ijcm} .2016 .72015$

11. Nicolson GL, Ash ME: Membrane Lipid Replacement for chronic illnesses, aging and cancer using oral glycerolphospholipid formulations with fructooligosaccharides to restore phospholipid function in cellular membranes, organelles, cells and tissues. Biochimica et Biophysica Acta Biomembranes 2017, 1859: 1704-1724. doi.org/10.1016/j.bbamem.2017.04.013

12. Küllenberg D, Taylor LA, Schneider M, Massing U: Health effects of dietary phospholipids. Lipids in Health and Disease, 2012, 11: e3. doi.org/10.1186/1476-511X-11-3

13. Nicolson GL, Rosenblatt S, Ferreira de Mattos G, Settineri R, Breeding PC, Ellithorpe RR, Ash ME: Clinical uses of Membrane Lipid Replacement supplements in restoring membrane function and reducing fatigue in chronic diseases and cancer. Discoveries 2016, 4(1): e54. doi10.15190/d.2016.1

14. van Meer G, Voelker DB, Feigenson GW: Membrane lipids: where they are and how they behave. Nature Reviews in Molecular and Cellular Biology 2008, 9: 112-124. doi.org/10.1038/nrm2330

15. Zierenberg O, Assmann G, Schmitz G, Rosseneu M: Effect of polyenephosphotidylcholine on cholesterol uptake by human high-density lipoprotein. Atherosclerosis 1981, 39(4): 527-542.doi.org/10.1016/0021-9150(81)90010-1

16. Dobbins WO III: Morphologic aspects of lipid absorption. American Journal of Clinical Nutrition 1969, 22: 257-265. doi.org/10.1093/ajcn/22.3.257

17. Zierenberg O, Grundy SM: Intestinal Absorption of Polyenephosphatidylcholine in Man. Journal of Lipid Research 1982, 23: 1136-1142.

18. Escribá PV: Membrane-lipid therapy: a new approach in molecular medicine. Trends in Molecular Medicine 2006, 12: 34-43. doi.org/10.1016/j.molmed.2005.11.004

19. Ibarguren M, López DJ, Escribá PV: The effect of natural and synthetic fatty acids on membrane structure, microdomain organization, cellular functions and human health. Biochimica et Biophysica Acta Biomembranes 2014, 1838: 1518-1528. doi.org/10.1016/j.bbamem.2013.12.021

20. Kroenke K, Wood DR, Mangelsdorff AD, Meiero NJ, Powell 
JB: Chronic fatigue in primary care. Prevalence, patient characteristics, and outcome. Journal of the American Medical Association 1988, 260; 929-934. doi.org/10.1001/jama.1988.03410070057028

21. Filler K, Lyon D, Bennett J, McCain N, Elswick R, Lukkahatai $\mathrm{N}$, Saligan LN: Association of mitochondrial dysfunction and fatigue: a review of the literature. BBA Clinical 2014, 1: 1223. doi.org/10.1016/j.bbacli.2014.04.001

22. Nicolson GL: Mitochondrial dysfunction and chronic disease: treatment with natural supplements. Integrative Medicine 2014, 13(4): 35-43

23. Komaroff AL, Buchwald D: Symptoms and signs of chronic fatigue syndrome. Reviews of Infectious Diseases 1991, 13(Suppl 1): S8-S11.

24. Komaroff AL, Fagioli LR, Geiger AM, Doolittle TH, Lee A, Kornish RJ, Gleit AB, et al.: An examination of the working case definition of chronic fatigue syndrome. American Journal of Medicine 1996, 100(1): 56-64 doi.org/10.1016/S0002-9343(96)90012-1

25. Nicolson GL: Lipid replacement therapy: a nutraceutical approach for reducing cancer-associated fatigue and the adverse effects of cancer therapy while restoring mitochondrial function. Cancer \& Metastasis Reviews 2010, 29(3): 543-552. doi.org/10.1007/s10555-010-9245-0

26. Agadjanyan M, Vasilevko V, Ghochikyan A, Berns P, Kesslak P, Settineri RA, Nicolson GL: Nutritional supplement (NT Factor) restores mitochondrial function and reduces moderately severe fatigue in aged subjects. Journal of Chronic Fatigue Syndrome 2003, 11(3): 23-36. doi.org/10.1300/J092v11n03 03

27. Nicolson GL, Ellithrope R: Lipid replacement and antioxidant nutritional therapy for restoring mitochondrial function and reducing fatigue in chronic fatigue syndrome and other fatiguing illnesses Journal of Chronic Fatigue Syndrome 2006, 13(1): 57-68. doi.org/10.1300/J092v13n01 06

28. Nicolson GL, Settineri R, Ellithorpe E: Lipid Replacement Therapy with a glycophospholipid formulation with NADH and CoQ10 significantly reduces fatigue in intractable chronic fatiguing illnesses and chronic Lyme disease. International Journal of Clinical Medicine 2012, 3(3): 164170.doi.org/10.4236/ijcm.2012.33034

29. Cernacchi T, Bertoldin T, Farina C, Flori MG. Crepaldi G: Cognitive decline in the elderly: a double-blind, placebo- controlled multicenter study on the efficacy of phosphatidylserine administration. Aging (Milano) 1993, 5: 123-133.

30. Jorissen BL, Brouns F, Van Boxtel MP, Ponds RW, Verhey FR, et al: The influence of soy-derived phosphatidylserine on cognition in age-associated memory impairment. Nutritional Neuroscience 2001, 4: 121-134. doi/abs/10.1080/1028415X.2001.11747356

31. Ellithorpe RA, Settineri R, Mitchell CA, Jacques B, Ellithorpe E, Nicolson GL: Lipid replacement therapy drink containing a glycophospholipid formulation rapidly and significantly reduces fatigue while improving energy and mental clarity. Functional Foods in Health and Disease 2011, 1(8): 245-254. DOI:10.31989/ffhd.v1i8.126

32. Nicolson GL, Settineri R, Ferreira G, Breeding P: Reduction of pain, fatigue, gastrointestinal and other symptoms and improvement in quality of life indicators in fibromyalgia patients with Membrane Lipid Replacement glycerolphospholipids and controlled-release caffeine. International Journal of Clinical Medicine 2018, 9: 560-579. doi.org/10.4236/ijcm.2018.97051

33. Nicolson GL, Breeding PC: Membrane Lipid Replacement for reduction of pain, fatigue, gastrointestinal and other symptoms in patients with peripheral pain: case reports. Case Reports \& Reviews 2020; 1(2): 1-3. DOI:10.33425/2693-1516.1007

34. Nicolson GL, Settineri R, Ellithorpe R: Neurodegenerative and fatiguing Illnesses, infections and mitochondrial dysfunction: use of natural supplements to restore mitochondrial function. Functional Foods in Health and Disease 2014, 4(1): 23-65. DOI:0.31989/ffhd.v4i1.26

35. Nicolson GL: Metabolic syndrome and mitochondrial function: molecular replacement and antioxidant supplements to prevent membrane oxidation and restore mitochondrial function. Journal of Cellular Biochemistry 2007, 100(6): 1352-1369. doi.org/10.1002/jcb.21247

36. Nygard O, Vollset SE, Refsum H, Stensvold I, Tverdal A, Nordrehaug JE, Ueland M, Kvale G: Total plasma homocysteine and cardiovascular risk profile: the Hordaland Homocysteine Study. JAMA 1995, 274: 1526-1533. doi.10.1001/jama.1995.03530190040032

37. Washio T, Nomoto K, Watanabe I, Tani S, Nagao K, Hirayama A: Relationship between plasma homoccysteine levels and 
congestive heart failure in patients with acute myocardial infarction. International Heart Journal 2011, 52: 224-228. doi.org/10.1536/ihj.52.224

38. Ellithorpe RR, Settineri R, Ellithorpe T, Nicolson GL: Blood homocysteine and fasting insulin levels are reduced and erythrocyte sedimentation rates are increased with a glycophospholipid-vitamin formulation: a retrospective study in older subjects. Functional Foods in Health and Disease 2015, 5(4): 126-135. DOI:10.31989/ffhd.v5i4.177

39. Campbell JN, Meyer RA: Mechanisms of neuropathic pain. Neuron 2006, 52(1): 77-96 doi.org:10.1016:j.neuron.2006.09.021

40. Staud R: Peripheral pain mechanisms in chronic widespread pain. Best Practice Research in Clinical Rheumatology 2011, 25: 155-164.doi.org/10.1016/j.berh.2010.01.010

41. Ferreira G, Costa C, Bassaizteguy V, Santos M, Cardozo R, Montes J, Settineri R, Nicolson GL: Incubation of human sperm with micelles made from glycerolphospholipid mixtures increases sperm motility and resistance to oxidative stress. PloS One 2018, 13(6): article e0197897. doi.org/10.1371/journal.pone.0197897

42. Taberner FJ, Fernandez-Ballester G, Fernandez-Carvajal A, Ferrer-Montiel A: TRP channels interaction with lipids and its implications in disease. Biochimica et Biophysica Acta Biomembranes 2015, 1848:1818-1827. doi.org/10.1016/j.bbamem.2015.03.022

43. Zhang $Y$, Chen X, Gueydan C, Han J: Plasma membrane changes during programmed cell death. Cell Research 2018, 28:9-21.doi.org/10.1038/cr.2017.133

44. Increased reactive oxygen species (ROS) formation and mitochondrial inner membrane lipid and protein oxidative damage with aging. From: Grimm A, Eckert A: Brain aging and neurodegeneration: from a mitochondrial point of view. Journal of Neurochemistry 2017; 143: 418-431 .doi.org/10.1111/inc.14037 